\title{
ESTUDO DA DESIDRATAÇÃO DE PÊSSEGOS POR TRATAMENTO OSMÓTICO E SECAGEM
}

\author{
JANESSA BUAES BOEIRA * \\ GUSTAVO BEULKE STRINGARI * * \\ JOÃO BORGES LAURINDO ***
}

\begin{abstract}
O objetivo deste trabalho foi estudar alternativas de processo para obtenção de pêssego desidratado (passas de pêssego) de boa qualidade. Investigouse o acoplamento de processos de pré-desidratação como a desidratação osmótica (DO) e a impregnação a vácuo (IV), com métodos de desidratação complementar (como a secagem em estufa e a aplicação de pulsos de vácuo). A desidratação de pêssegos pela combinação de DO, secagem por pulsos de vácuo e estufa mostrou-se mais adequada para obtenção de frutas secas que a simples desidratação dos pêssegos in natura em estufa. A IV não constituiu boa alternativa para a desidratação, devido à baixa porosidade do pêssego. Este trabalho mostrou a possibilidade de se obter pêssegos desidratados de boa qualidade a partir da escolha de alternativas de processo adequadas.
\end{abstract}

PALAVRAS-CHAVE: DESIDRATAÇÃO OSMÓTICA; Prunus pérsica; SECAGEM; PÊSSEGOS.

* $\quad$ Mestre em Engenharia de Alimentos, Laboratório de Propriedades Físicas dos Alimentos, Universidade Federal de Santa Catarina (UFSC), Florianópolis, SC (e-mail: janessab@terra.com.br).

** Aluno de Iniciação Científica, Curso de Engenharia de Alimentos, Laboratório de Propriedades Físicas dos Alimentos, UFSC, Florianópolis, SC (e-mail: engenheirogus@gmail.com).

*** Professor, Doutor, Departamento de Engenharia Química e Engenharia de Alimentos, UFSC, Florianópolis, SC. (e-mail: joao@enq.ufsc.br). 


\section{INTRODUÇÃO}

O pêssego (prunus pérsica) fruto originário da China, é consumido em todo o mundo. A produção mundial anual de pêssegos alcança 11 milhões de toneladas, segundo dados da FAO (2005), sendo o Brasil um dos dez maiores produtores. A produção nacional de pêssegos é de 200.000 toneladas por ano (IBGE, 2004) e está concentrada principalmente nos estados da região sul, notadamente no Rio Grande do Sul. A oferta de pêssegos in natura no Brasil ocorre nos meses de novembro a março. Os pêssegos também são consumidos durante o ano todo na forma de sucos, doces, geléias, compotas e adicionados aos iogurtes. No entanto, não se produz pêssego desidratado em escala comercial no Brasil. Considerando o alto valor agregado das frutas secas encontradas no mercado e o fato de parte importante das mesmas serem importadas, a alternativa de se obter novas frutas secas precisa de novos dados e estudos. Sendo fruto climatérico, o pêssego tem metabolismo acelerado e gera perdas pós-colheita que alcançam de 30 a $50 \%$ da produção (DI RIENZO, 2001). Embora o Brasil seja o terceiro maior produtor mundial de frutos in natura (SEBRAE, 2005), importa 172.000 toneladas de frutas desidratadas e exporta apenas 12.000 toneladas (FAO, 2005). A desidratação de pêssegos pode contribuir para a minimização das perdas e possibilitar agregação de valor ao fruto.

A desidratação osmótica (DO), processo utilizado para a remoção parcial da água dos alimentos (como frutas), consiste na sua imersão em solução com alta pressão osmótica e baixa atividade de água (RAOULT-WACK, 1994), ADO tem recebido grande atenção devido à possibilidade da utilização de temperaturas mais baixas, o que é conveniente para produtos termossensíveis. $O$ uso de temperaturas brandas evita danos à textura, cor e ao sabor, obtendo-se produto final com boa qualidade (PANAGIOTOU, KARATHANOS e MAROULIS, 1999). Além disso, trata-se de processo com baixo consumo de energia pela ausência de mudança de fase (RAOULT-WACK, 1994; RASTOGI et al., 2002).

A DO vem sendo utilizada como pré-tratamento, precedendo processos como o congelamento (HAWKES e FLINK, 1978; TALENS et al., 2003), a secagem a vácuo (KROKIDA et al. 2000), ou a secagem tradicional (RASTOGI et al., 2002; PARK, BIN e BROD, 2002). A DO atua na redução da atividade de água dos alimentos, inibindo o crescimento microbiano (RASTOGI et al., 2002), reduzindo o escurecimento enzimático e possibilitando o uso de menores concentrações de $\mathrm{SO}_{2}$ (POINTING, 1973; REPPA et al., 1999). Além disso, pode aumentar o tempo de vida-deprateleira, reduzir a perda de aromas em produtos secos ou com umidade intermediária e reduzir as mudanças de textura provocadas pelo congelamento (MAVROUDIS, GEKAS e SJÖHOLM, 1998).

$\mathrm{Na}$ DO de vegetais, a complexa estrutura da parede celular atua como membrana semipermeável que não é completamente seletiva (KOWALSKA e LENART, 2001) permitindo dois fluxos principais de transferência de massa. Em razão da diferença entre o potencial químico da água na solução externa e no produto ocorre fluxo de água do alimento para a solução. Por outro lado, devido à estrutura aberta do tecido celular nos espaços intercelulares ocorre a difusão do soluto da solução externa para o alimento (RAOULT-WACK, 1994). Mudanças estruturais no alimento também podem ocorrer durante a DO, como a deformação das células, alterações fisiológicas e a perda de solutos nativos do produto para a solução (RAOULT-WACK, 1994; CHIRALT e FITO, 2003; CHIRALT e TALENS, 2005). Esses fenômenos provocam mudanças nas propriedades macroscópicas do alimento, bem como nas propriedades ópticas e mecânicas, relacionadas com a aparência e com a textura, respectivamente (TORREGGIANI e BERTOLO, 2001; TALENS et al., 2002). Muitos autores estudaram as variáveis que influenciam a cinética da desidratação osmótica de frutas e vegetais, como o tipo de soluto, a composição da solução (HAWKES e FLINK, 1978), a temperatura da solução (OZEN et al., 2002; MADAMBA e LOPEZ, 2002), a concentração da solução (HAWKES e FLINK, 1978; OZEN et al., 2002; MADAMBA e LOPEZ, 2002), o tempo de processo (OZEN et al., 2002; MADAMBA e LOPEZ, 2002), a geometria das amostras (MADAMBA e LOPEZ, 
2002) e o nível de agitação da solução osmótica (MAVROUDIS, GEKAS e SJÖHOLM, 1998).

Diversas estratégias têm sido estudadas com o objetivo de se aumentar a transferência de massa durante a DO, tais como a elevação da temperatura da solução, maior concentração da solução, ou o aumento do tempo de processo. Entretanto, todas essas estratégias são limitadas, uma vez que provocam mudanças indesejáveis no aroma, cor e textura do alimento (SHI e FITO, 1994). Vários estudos têm demonstrado que o uso de vácuo na desidratação osmótica resulta em aumento na taxa de perda de água e ganho de sólidos (ZOZULEVICH e D'YACHENKO, 1969; SHI e FITO, 1994; FITO e PASTOR, 1994; MARTÍNEZ-MONZO et al., 1998; MÚJICA-PAZ et al., 2003 ab).

GALLI et al. (1996) avaliaram a influência da composição da solução osmótica nas características físico-químicas de pêssegos (Magno) tipo passa, utilizando soluções de sacarose e xarope de glicose de milho. Os resultados não apresentaram significância estatística sobre as características físico-químicas dos pêssegos tipo passa.

A secagem convencional com ar quente constitui processo de transferência de calor e massa com mudança de fase. Os fatores que governam os mecanismos de transferência determinam a taxa de secagem. Esses fatores são a pressão de vapor do material e do ar de secagem, a velocidade e temperatura do ar, a difusão da água no material, a espessura do material e a área superficial do material exposta ao ar de secagem (VAN ARSDEL, COPLEY e MORGAN, 1973).

A secagem usando microondas como fonte de energia representa alternativa que tem como vantagem a possibilidade de controle do aquecimento e a eficiência do uso da energia (SCHIFFMANN, 2001). Os pontos negativos envolvem o aquecimento desigual que ocorre em geometrias com partes finas e pontas, os danos na textura do alimento no caso de elevadas taxas de evaporação e o alto custo do equipamento (SUMNU, TURABI e OZPOT, 2005).

O objetivo deste trabalho foi estudar alternativas de processo para obtenção de passas de pêssego com qualidade, mediante acoplamento de processos de pré-desidratação (como a desidratação osmótica e impregnação a vácuo de soluções de açúcar) com métodos de desidratação complementares (como a secagem em estufa e a secagem utilizando sucessivos aquecimentos seguidos da aplicação de pulsos de vácuo).

\section{MATERIAL E MÉTODOS}

Os experimentos de desidratação osmótica foram realizados com pêssegos nacionais da variedade Marli e com pêssegos chilenos da variedade Springcrest. As amostras foram selecionadas pelo grau de maturação, por meio da medida do conteúdo de sólidos solúveis $\left({ }^{\circ}\right.$ Brix) e da resistência à penetração. Os pêssegos foram cortados ao meio e descascados manualmente, evitando diferenças nas propriedades estruturais devido à orientação celular (VINCENT, 1989).

\subsection{CARACTERIZAÇÃO FÍSICO-QUÍMICA DOS PÊSSEGOS IN NATURA}

O conteúdo de umidade foi determinado pela secagem a temperatura de $104^{\circ} \mathrm{C}$. As pesagens foram realizadas em balança semi-analítica, com precisão de 0,001 g (Tecnal, Linha 6K, Brasil). Para determinação da concentração média de sólidos solúveis utilizou-se refratômetro óptico manual com resolução de 0,2 ${ }^{\circ}$ Brix (Greers Ferry Glass, REF 107, Quitman, Arkansas, USA). Essas determinações seguiram as normas do IAL (1985).

A densidade aparente e a densidade real do pêssego foram determinadas por picnometria (SALVATORI et al., 1998a). Determinou-se a densidade aparente pela medida do volume de líquido deslocado pela amostra. A densidade real foi determinada com a amostra previamente triturada, homogeneizada e desaerada (pressão de $195 \mathrm{mmHg}=26 \mathrm{kPa}$, por $2 \mathrm{~h}$ ). Todas as análises foram feitas em triplicata. 
Calculou-se a porosidade das amostras por meio dos valores das densidades real e aparente de acordo com a Equação 1:

$$
\varepsilon=1-\frac{\rho_{b}}{\rho_{s}}
$$

Na qual: $\rho_{s}=$ densidade real da partícula e $\rho_{b}=$ densidade aparente.

Efetuou-se a determinação da atividade de água $\left(\mathrm{a}_{\mathrm{w}}\right)$ do pêssego in natura usando higrômetro (Aqualab Model Series 3, Decagon Devices Inc., Pullman, USA). A resistência à penetração foi avaliada com penetrômetro (Effegi Mod FT 327, $\varnothing=8 \mathrm{~mm}$ ), considerando-se verdes os pêssegos que resistiram à força maior ou igual a $1,5 \mathrm{kgf}$ e maduros os que resistiram à força menor ou igual a $0,5 \mathrm{kgf}$.

\subsection{ALTERNATIVAS INVESTIGADAS PARA PRODUÇÃO DA PASSA DE PÊSSEGO}

As alternativas investigadas para a obtenção de frutas desidratadas com boas características de textura estão esquematizadas na Figura 1. No primeiro caso realizou-se a secagem de frutas in natura em estufa com circulação de ar e renovação de ar a temperatura de $60^{\circ} \mathrm{C}$. $\mathrm{O}$ mesmo procedimento de secagem foi aplicado para frutas pré-desidratadas por desidratação osmótica (DO). Outro tratamento de desidratação e modificação de textura por pulso de vácuo também foi estudado para as frutas tratadas por DO. No entanto, esse tratamento não dispensou o uso da secagem em estufa como método de secagem final. No caso do pré-tratamento das frutas por impregnação a vácuo (IV) optou-se por não realizar a secagem complementar.

\section{FIGURA 1 - FLUXOGRAMA DAS ALTERNATIVAS AVALIADAS PARA A OBTENÇÃO DO PÊSSEGO DESIDRATADO}

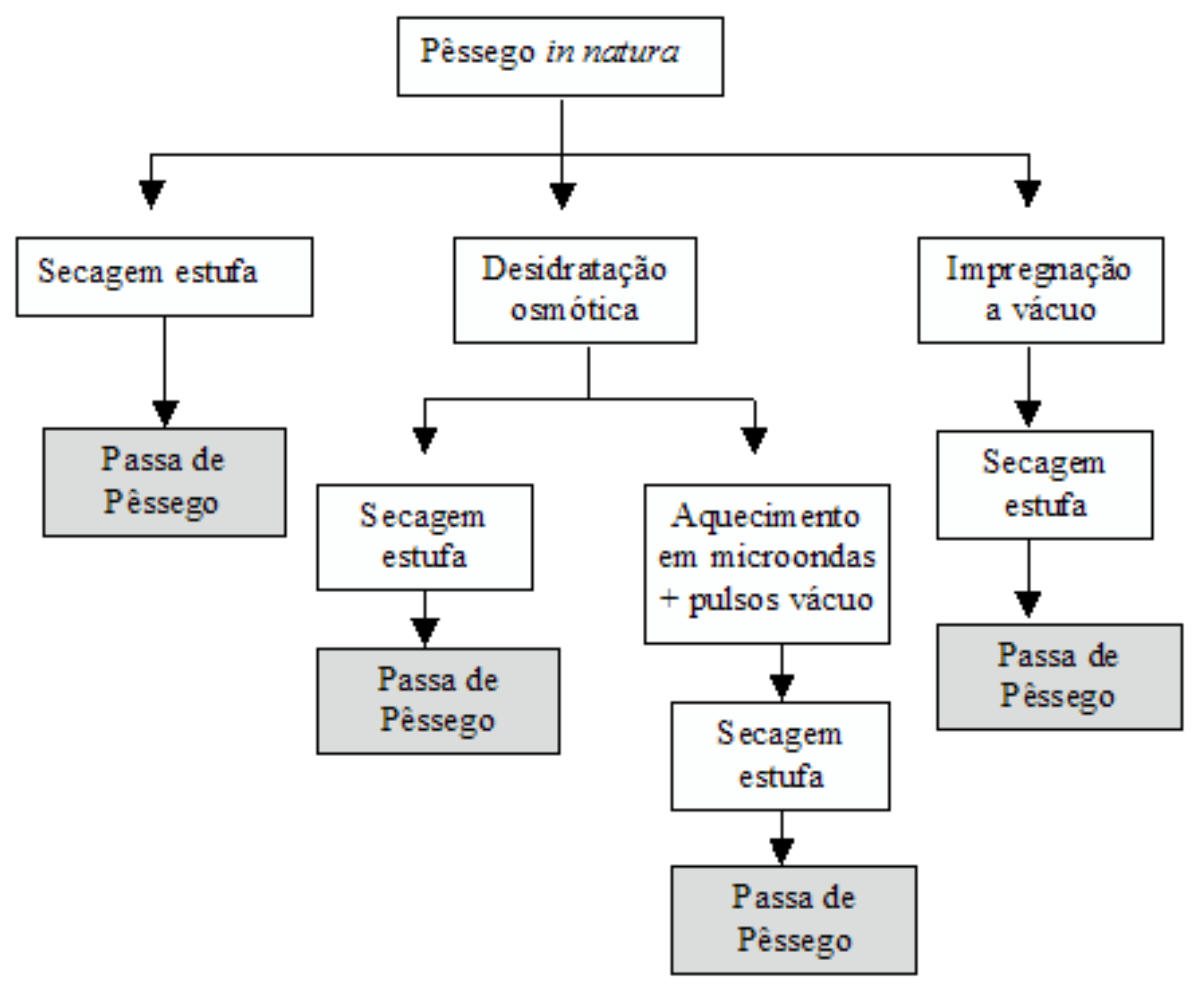


O dispositivo utilizado nos experimentos de desidratação osmótica e de impregnação a vácuo de pêssegos está esquematizado na Figura 2. Consistiu de câmara encamisada (volume interno de 13,35 L) com controle de temperatura realizado pela água circulante, proveniente de banho termostatizado (Microquímica, Modelo MQBMP-01). As amostras foram mantidas submersas na solução por tela plástica para evitar que as mesmas flutuassem.

Para estudar a efetividade da aplicação de vácuo na desidratação osmótica instalou-se linha de vácuo junto ao dispositivo, usando bomba de vácuo (Boc Edwards, Modelo D-LAB 10-8), armadilha para gotículas (trapping) e vacuômetro digital (lope, Modelo TWPI), visando o monitoramento online da pressão da câmara. $O$ sistema permitiu a obtenção de vácuo máximo de 95,7 kPa (pressão absoluta de 5,3 kPa) na câmara de vácuo.

\section{FIGURA 2 - DISPOSITIVO EXPERIMENTAL UTILIZADO PARA ESTUDOS \\ DE DESIDRATAÇÃO OSMÓTICA E DE IMPREGNAÇÃO \\ A VÁCUO DE PÊSSEGOS}

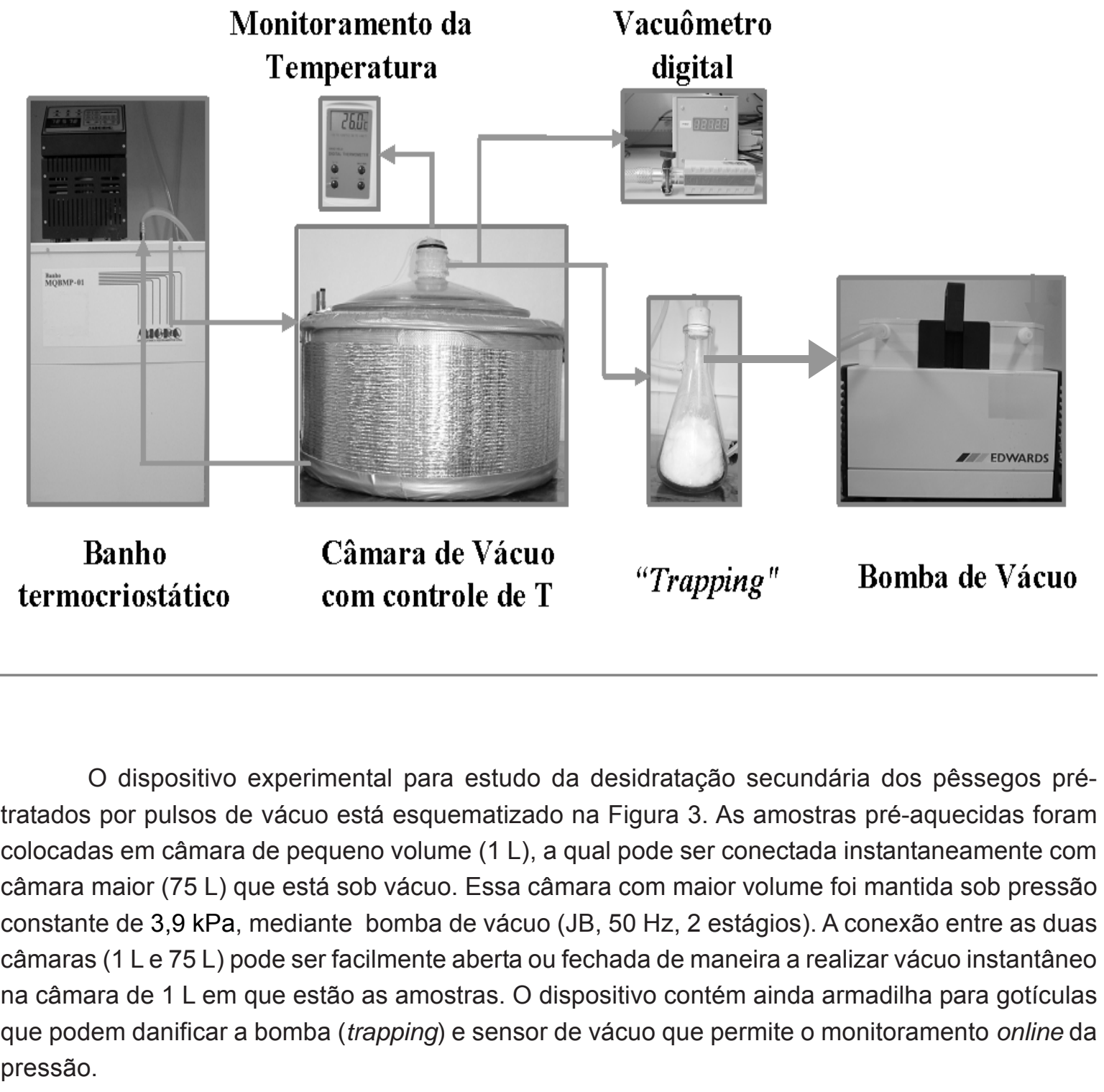




\section{FIGURA 3 - DISPOSITIVO EXPERIMENTAL UTILIZADO PARA APLICAÇÃO} DOS PULSOS DE VÁCUO

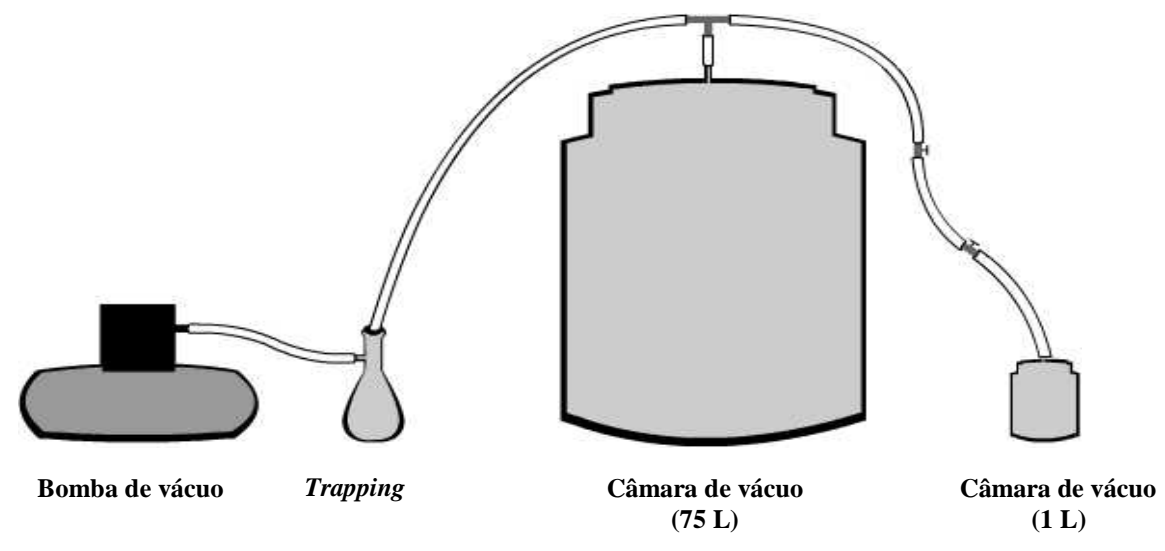

Realizou-se o cálculo da perda de água (WL) e do ganho de sólidos (SG) com base nas equações 2 e 3 :

$$
\begin{array}{r}
W L=\frac{w_{w 0}-w_{w}}{w_{0}} \cdot 100 \\
S G=\frac{w_{s}-w_{s o}}{w_{0}} \cdot 100
\end{array}
$$

Nas quais:

$\mathrm{w}_{\mathrm{wo}}=$ conteúdo inicial de água na amostra;

$\mathrm{w}_{\mathrm{w}}=$ conteúdo de água na amostra ao fim do tratamento;

$\mathrm{w}_{\mathrm{o}}=$ massa inicial da amostra;

$\mathrm{w}_{\mathrm{s}}=$ massa de sólidos secos ao fim do tratamento; e

$\mathrm{w}_{\mathrm{so}}=$ massa inicial de sólidos secos da amostra .

Obteve-se a massa inicial da amostra pela pesagem em balança semi-analítica, enquanto o conteúdo inicial de água e de sólidos secos foi calculado após determinação da umidade inicial da amostra. A massa final da amostra também foi obtida por pesagem após o processo de DO, sendo o conteúdo de água e de sólidos secos novamente calculados após a determinação da umidade (no caso da amostra já tratada).

Para verificar a eficácia da aplicação do vácuo em alimentos com baixa porosidade (como é o caso do pêssego), os parâmetros SG e WL decorrentes de experimentos de desidratação osmótica a pressão atmosférica e de impregnação a vácuo de oito amostras foram comparados.

Os experimentos com aplicação de vácuo foram caracterizados pela etapa com pressão de $5,3 \mathrm{kPa}$ durante $20 \mathrm{~min}$, seguida por outra etapa de 80 min a pressão atmosférica. A concentração da solução de sacarose foi de $65^{\circ}$ Brix, mantida na temperatura de $30^{\circ} \mathrm{C}$.

Os experimentos a pressão atmosférica (DO) e a etapa a pressão atmosférica do processo de impregnação a vácuo (após a aplicação de vácuo) foram realizados nas mesmas condições. As amostras submetidas à DO foram deixadas em pressão atmosférica por 100 min, garantindo assim o mesmo tempo de contato global entre as amostras e a solução de sacarose para os dois processos.

Para verificar a influência da temperatura na DO, dez amostras de pêssegos foram submersas em solução com concentração de sacarose igual a $65^{\circ}$ Brix durante 45 min. Esse experimento foi 
realizado nas temperaturas constantes de $30,40,50$ e $60^{\circ} \mathrm{C}$, sendo os parâmetros WL e SG avaliados.

Determinou-se cinética de desidratação osmótica, mediante experimentos com tempos de contato amostras-solução de $0.5,1,2,4,6,10,24$ e 48h. Dez amostras de pêssegos para cada experimento foram submersas em solução osmótica de sacarose de $65^{\circ}$ Brix, mantida em temperatura constante de $40^{\circ} \mathrm{C}$. As evoluções temporais dos parâmetros WL e SG foram determinadas para cada experimento.

Para estudar a influência do grau de maturação dos pêssegos na desidratação osmótica, frutas verdes e maduras foram selecionadas e seu grau de maturação determinado pela concentração de sólidos solúveis e pelo uso do penetrômetro (conforme descrito anteriormente). Dez amostras de pêssegos verdes e dez amostras de pêssegos maduros foram submersas separadamente em solução de sacarose com concentração de $65^{\circ}$ Brix e temperatura constante de $40^{\circ} \mathrm{C}$. A solução de sacarose foi agitada durante todo experimento por bomba submersa (Atman AT-010) com vazão nominal de $1200 \mathrm{~L} / \mathrm{h}$ (bombeamento de água). No bombeamento das soluções de sacarose ocorreu redução da vazão de bombeamento para até um terço dessa vazão nominal, mas a agitação da solução manteve-se em níveis satisfatórios. Os experimentos foram realizados para tempos de 10, 24 e 48h, sendo os parâmetros WL e SG determinados para cada ensaio.

\subsection{AVALIAÇÃO EMPÍRICA DA SECAGEM COMPLEMENTAR MEDIANTE CURVAS DE SECAGEM}

Utilizaram-se amostras de pêssego, previamente tratadas por DO com solução de sacarose de $65^{\circ} \mathrm{Brix}$ a $40^{\circ} \mathrm{C}$, durante $24 \mathrm{~h}$. Parte das amostras sofreu aplicações de vácuo instantâneo antes da secagem em estufa. $O$ tratamento consistiu na seqüência de três aquecimentos durante $15 \mathrm{~s}$ em forno de microondas comercial, seguidos da aplicação de vácuo instantâneo. A secagem em estufa com circulação forçada de ar ocorreu a $60^{\circ} \mathrm{C}$, que se enquadra na faixa indicada para secagem de frutas (SCHULTZ et al., 2006). As amostras que sofreram aplicações de pulsos de vácuo e as amostras que não sofreram essas aplicações foram analisadas distintamente, tendo seus valores de umidade sido determinados ao longo da secagem. Esses estudos foram completamente empíricos e preliminares, pois não se dispunha de equipamento de microondas com potência calibrada e nem de sensores de temperatura de fibra ótica (para usar no interior do forno de microondas). Os dados da potência dissipada pelo forno de microondas, da quantidade absorvida pelos pêssegos e a evolução da temperatura são fundamentais para o tratamento fenomenológico dos resultados.

\section{RESULTADOS E DISCUSSÃO}

Os pêssegos da variedade Springcrest apresentaram menor densidade e maior concentração de sólidos solúveis em relação aos pêssegos da variedade Marli (Tabela 1). No entanto, os valores de umidade, porosidade e atividade de água foram semelhantes para ambas as variedades.

\section{TABELA 1 - CARACTERIZAÇÃO FÍSICO-QUÍMICA DOS PÊSSEGOS MARLI E SPRINGCREST IN NATURA}

\begin{tabular}{lcc}
\hline Parâmetros & Marli & Springcrest \\
\hline Conteúdo de Umidade (kg água/kg amostra) & $0,898 \pm 0,021$ & $0,893 \pm 0,010$ \\
Sólidos Solúveis Totais ( $\left.{ }^{\circ} \mathrm{Brix}\right)$ & $12,3 \pm 0,7$ & $13,5 \pm 0,3$ \\
Densidade Aparente $(\mathrm{kg} / \mathrm{m} 3)$ & $981 \pm 8$ & $954 \pm 7$ \\
Densidade Real $(\mathrm{kg} / \mathrm{m} 3)$ & $1035 \pm 1$ & $1006 \pm 2$ \\
Porosidade $(\varepsilon)$ & $0,052 \pm 0,008$ & $0,052 \pm 0,008$ \\
Atividade de Água $\left(\mathrm{a}_{\mathrm{w}}\right)$ & $0,994 \pm 0,001$ & $0,995 \pm 0,001$ \\
\hline
\end{tabular}




\subsection{EFICÁCIA DA APLICAÇÃO DE VÁCUO NA DESIDRATAÇÃO OSMÓTICA}

Na Figura 4 consta a comparação entre os valores de WL e SG na DO e na IV para o mesmo tempo de contato entre as amostras e a solução de açúcar. Os resultados indicaram que a utilização do vácuo exerceu influência na quantidade de solução impregnada no pêssego. O ganho de sólidos (SG) foi maior quando a etapa inicial de vácuo foi utilizada, devido à degaseificação da matriz porosa (FITO e PASTOR, 1994). Entretanto, a entrada da solução resultou em perda de água (WL) menor e aumento da umidade final do produto. Dessa forma, a aplicação de vácuo em processo de DO torna-se interessante quando o objetivo é impregnar determinada solução e não para desidratar o pêssego. Para outras frutas mais porosas (como diferentes variedades de maçãs), a impregnação a vácuo pode sob determinadas condições cumprir os dois objetivos.

Autilização do vácuo para a obtenção de pêssego desidratado não seria alternativa interessante, tanto pela menor perda de água quanto pelo pequeno aumento no ganho de sólidos. Isso implica em aumento dos custos operacionais, sem ganhos efetivos na eficiência do processo. Esse comportamento pode ser explicado pela baixa porosidade do pêssego (aproximadamente 5\%), que diminui a influência da aplicação do vácuo.

\section{FIGURA 4 - GANHO DE SÓLIDOS (SG) E PERDA DE ÁGUA (WL) NA DO (100 min) E IV (20 min DE VÁCUO E 80 min A PRESSÃO ATMOSFÉRICA) DE PÊSSEGOS}

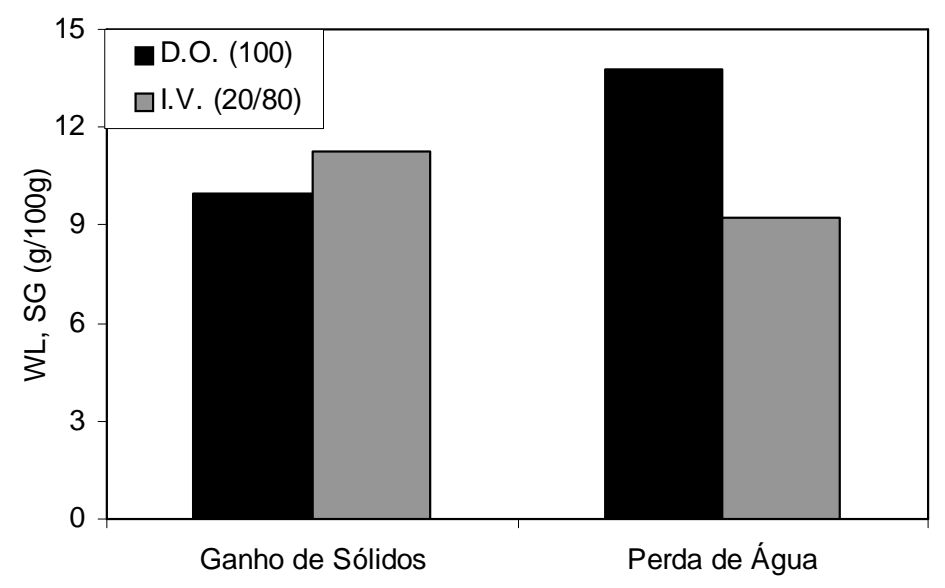

Condições do ensaio: $30^{\circ} \mathrm{C}$, concentração da solução de $65^{\circ}$ Brix, relação fruta:solução de 1:30.

\subsection{INFLUÊNCIA DA TEMPERATURA NA DESIDATAÇÃO OSMÓTICA}

Os resultados experimentais indicaram que a temperatura de processo exerceu grande influência na perda de água das amostras de pêssego (Figura 5). No entanto, não foi verificada influência da temperatura para o ganho de sólidos. SILVEIRA, RAHMAN e BICIG (1996), também constataram que elevadas temperaturas aumentaram as taxas de perda de água, mas não influenciaram o ganho de sólidos de abacaxis submetidos à desidratação osmótica.

A elevação da temperatura aumentou a eficiência da desidratação, mas dificultou a manipulação do produto que se tornou muito mais frágil. O problema mostrou-se mais evidente quando se utilizou temperatura de $60^{\circ} \mathrm{C}$. Dificuldades semelhantes foram citadas para desidratação osmótica de kiwi em temperaturas iguais ou superiores a $50^{\circ} \mathrm{C}$ (VIAL, GUILBERT e CUQ, 1991) e de papaia na temperatura de $60^{\circ} \mathrm{C}$ (HENG, GUILBERT e CUQ, 1990). Ambas as frutas apresentaram modificações indesejáveis quanto à coloração, textura e valor nutricional. No presente caso, a temperatura de $40^{\circ} \mathrm{C}$ mostrou-se adequada em relação aos aspectos de cinética de desidratação e preservação da textura. 


\section{FIGURA 5 - INFLUÊNCIA DA TEMPERATURA NA PERDA DE ÁGUA E NO GANHO DE SÓLIDOS NA DESIDRATAÇÃO OSMÓTICA DE PÊSSEGOS}

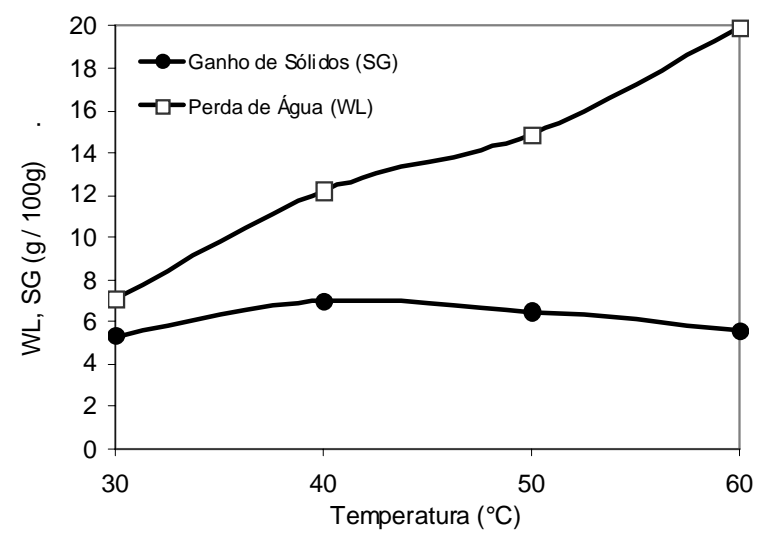

Condições de ensaio: 45 min, concentração da solução de $65^{\circ}$ Brix, relação fruta:solução de 1:30.

\subsection{CINÉTICA DA DESIDRATAÇÃO OSMÓTICA}

Conforme apresentado na Figura 6, a maior parte do ganho de sólidos ocorre nos primeiros 30 min de DO. Se comparado com o valor máximo de SG (constatado no tempo de 6 horas) verifica-se que $63,7 \%$ do ganho em sólidos ocorreu nos primeiros 30 min. Após atingir esse valor máximo no tempo de 6 horas, o pêssego passa a perder sólidos, provavelmente, devido à expulsão da solução osmótica dos poros da fruta com a diminuição do volume das amostras (encolhimento).

Verificou-se que a WL ocorreu sempre de maneira progressiva, mas também constatou-se fase inicial em que a cinética de perda de água é maior. Se comparada à perda de água total em 48 horas observa-se que $77,3 \%$ da perda ocorre nas primeiras 10 horas, enquanto que $90,6 \%$ da água é perdida no primeiro dia (24 horas). Desse modo, recomenda-se que o processo de DO de pêssegos (em metades) seja realizado com tempos de contato fruta-solução osmótica de 10 a 24 horas.

\section{FIGURA 6 - CINÉTICA DO GANHO DE SÓLIDOS (SG) E PERDA DE ÁGUA (WL) NA DESIDRATAÇÃO OSMÓTICA DE PÊSSEGOS}

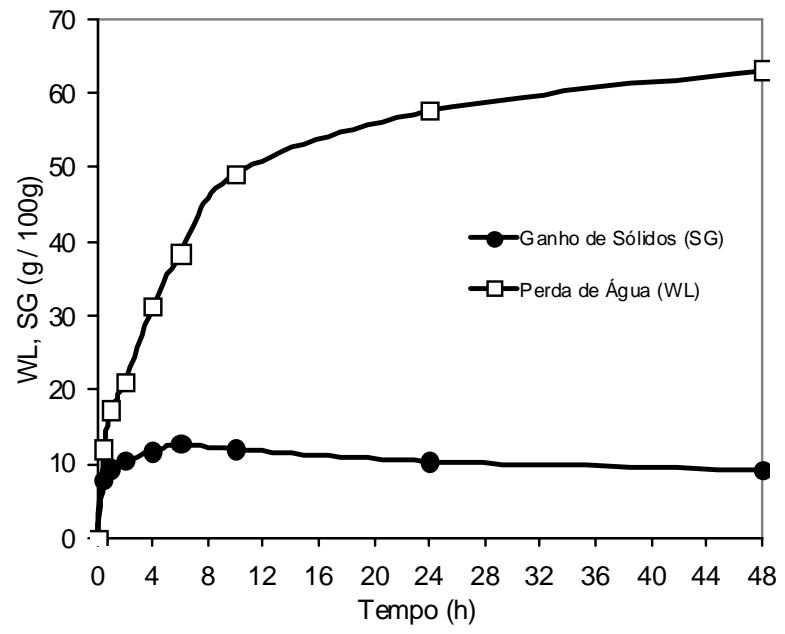

Condições de ensaio: $40^{\circ} \mathrm{C}$, concentração da solução de $65^{\circ}$ Brix, relação fruta:solução de 1:30. 


\subsection{INFLUÊNCIA DO GRAU DE MATURAÇÃO}

Na Figura 7 são apresentados os resultados da influência do grau de maturação (pêssegos verdes/maduros) na perda de água e ganho de sólidos durante a DO. Os pêssegos maduros apresentaram maior tendência para ganhar sólidos do que os pêssegos verdes, não sendo observada diferença entre os dois graus de maturação para a perda de água.

O maior ganho de sólidos quando se utiliza pêssegos maduros pode estar relacionado com a perda de seletividade da membrana da célula vegetal (devido ao estágio avançado de maturação), o que facilitaria o transporte do açúcar para dentro das células por difusão. Para ambos os casos (pêssegos verdes e maduros), o tempo de 24 horas evidenciou o maior ganho de sólidos. Observouse também que a maior parte da perda de água ocorreu nas primeiras $24 \mathrm{~h}$ do processo. Se comparados aos valores totais da perda de água após $48 \mathrm{~h}$ de DO, a perda de água após 24h representou $94,7 \%$ do total perdido no caso dos pêssegos verdes e $90,8 \%$ no caso dos pêssegos maduros.

\section{FIGURA 7 - INFLUÊNCIA DO GRAU DE MATURAÇÃO NA PERDA DE ÁGUA E NO GANHO DE SÓLIDOS NA DESIDRATAÇÃO OSMÓTICA DE PÊSSEGOS}

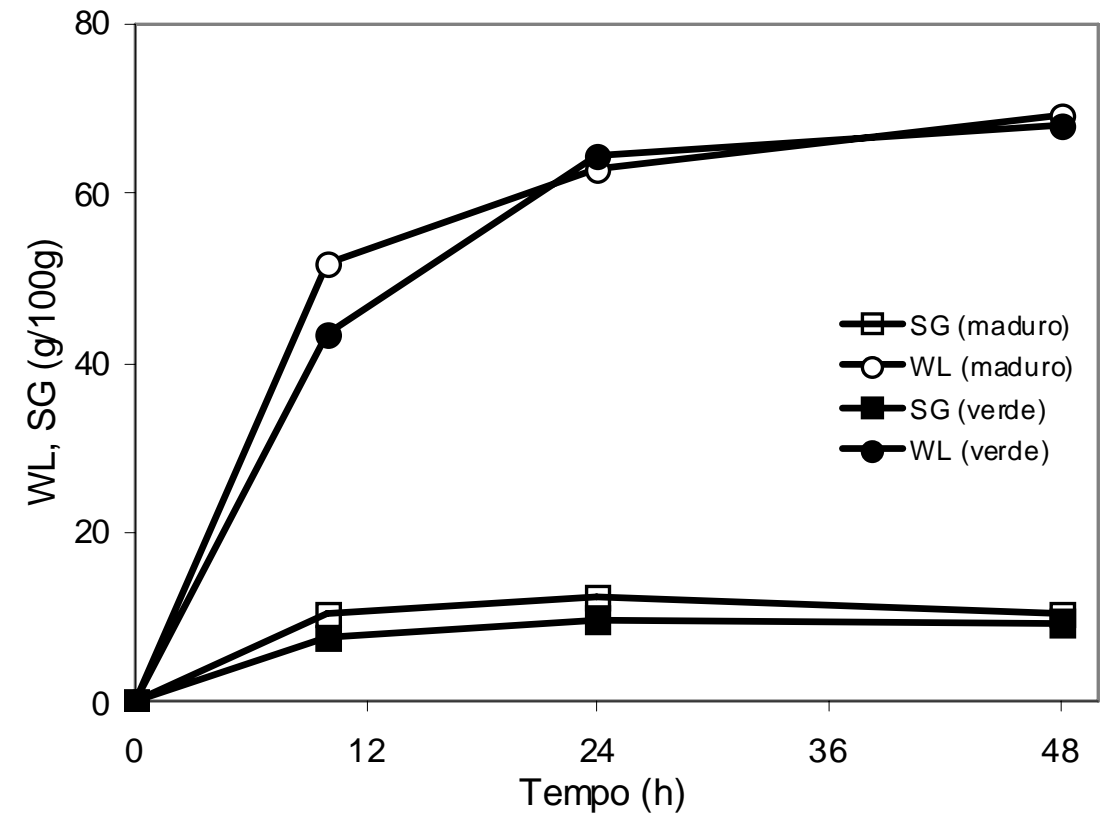

Condições de ensaio: $40^{\circ} \mathrm{C}$ e concentração da solução $65^{\circ}$ Brix.

\subsection{AVALIAÇÃO DA SECAGEM COMPLEMENTAR - CURVAS DE SECAGEM}

As amostras que sofreram apenas DO (secagem A) iniciaram a secagem em estufa com 1,331 g água/g de sólidos secos, enquanto as amostras que sofreram o tratamento de DO seguido por três pulsos de vácuo instantâneo (secagem B) entraram na estufa com umidade igual a 0,984 $\mathrm{g}$ água/g de sólidos secos. A secagem foi interrompida no momento em que as amostras atingiram atividade de água $\left(a_{w}\right)$ próxima de 0,7 , considerada adequada por analogia com os valores médios 
de $a_{w}$ de ameixas e damascos desidratados encontrados no comércio $\left(a_{w}\right.$ de 0,725 e 0,730, respectivamente). No caso da secagem $A$, as amostras atingiram $a_{w}$ de 0,705 somente com umidade igual a 0,242 g de água/g de sólidos secos, o que ocorreu após 25 horas de secagem. No caso da secagem $B$, as amostras atingiram $a_{w}$ de 0,710 com umidade de $0,368 \mathrm{~g}$ de água/g de sólidos secos em apenas 13 horas de secagem em estufa.

A aplicação dos pulsos de vácuo, que antecedeu a secagem B, trouxe dois benefícios evidentes. O primeiro foi a diminuição do tempo de secagem em estufa para atingir a mesma umidade. Essa diminuição ocorreu em razão da amostra iniciar a secagem com menor teor de umidade e pelo fato dos pulsos de vácuo favorecerem a formação de estrutura porosa, facilitando a secagem (DROUZAS e SCHUBERT, 1996). O segundo benefício envolveu a possibilidade de atingir a mesma $\mathrm{a}_{\mathrm{w}}$ mantendo maior umidade no produto, o que pode representar melhores propriedades de textura para as frutas desidratadas.

Na Figura 8 são apresentadas as taxas de secagem (g água evaporada/h) em estufa das amostras tratadas pelos processos $\mathrm{A}$ e $\mathrm{B}$. $\mathrm{Na}$ amostra submetida ao processo $\mathrm{B}$ percebe-se período de taxa de secagem constante nas duas primeiras horas, o que não é evidenciado no processo $A$. Isso provavelmente ocorreu pelo fato das amostras submetidas aos pulsos de vácuo se apresentarem mais porosas. Os picos evidenciados na nona hora de secagem pelo processo $B$ e na décima sétima hora de secagem do processo A ocorreram após a virada das amostras, ou seja, a inversão das faces apoiadas na base da placa no interior da estufa.

\section{FIGURA 8 - CURVA DA TAXA DE SECAGEM (G/H) DAS AMOSTRAS SUBMETIDAS AOS PROCESSOS A E B}

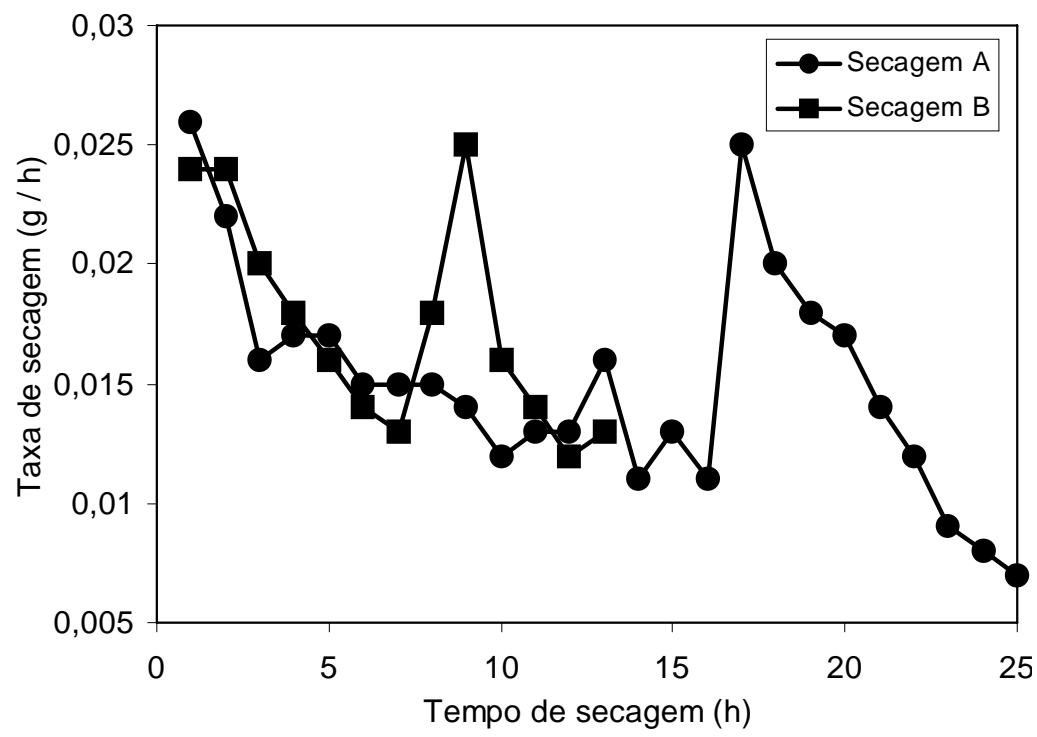

\section{CONCLUSÃO}

O processo de desidratação de pêssegos pela combinação de DO e secagem por pulsos de vácuo e estufa é adequado para a obtenção de pêssegos secos, mas a aplicação da impregnação a vácuo não se apresenta como alternativa viável para a desidratação devido à baixa porosidade do pêssego.

Este trabalho mostrou a possibilidade de se obter frutos desidratados de boa qualidade a partir da escolha de alternativas de processo adequadas. 


\begin{abstract}
STUDY OF PEACHES DEHYDRATION BY OSMOTIC TREATMENT AND DRYING

The purpose of this work was to investigate processes alternatives for obtaining dehydrated peach presenting good quality. For that the coupling of pre-dehydration processes, the osmotic dehydration (OD) and the vacuum impregnation ( $\mathrm{VI}$ ), with complementary dehydration (convective drying and the application of vacuum pulses) was investigated. The dehydration of peach by the combination of OD, drying by vacuum pulses and convective drying in oven was the best alternative to obtain dried fruits. The application of VI was not a good alternative to dehydration, due to the low peaches porosity. This work demonstrated that is possible to produce dehydrated peaches of good quality selecting appropriate processes.
\end{abstract}

KEY-WORDS: OSMOTIC DEHYDRATION; Prunus pércica; DRYING; PEACHES.

\title{
REFERÊNCIAS
}

1 CHIRALT, A.; FITO, P. Transport mechanisms in osmotic dehydration: the role of the structure. Food Science and Technology International, v. 9, p. 179-186, 2003.

2 CHIRALT, A .; TALENS, P. Physical and chemical changes induced by osmotic dehydration in plant tissues. Journal of Food Engineering, v. 67, p. 167-177, 2005.

3 DI RIENZO, C. A importância das câmaras frias na horticultura. Tecnologia da Refrigeração, n. 5, p. 1622, 2001.

4 DROUZAS, A. E.; SCHUBERT, H. Microwave application in vacuum drying of fruits. Journal of Food Engineering, v. 28, p. 203-209, 1996.

5 FAO. Food and Agriculture Organization of the United Nations. FAO Statistical Databasis. Disponível em: http:// www.todafruta.com.br. Acesso em: 12 mar.2005.

6 FITO, P.; PASTOR, R. Non-diffusional mechanisms occurring during vacuum osmotic dehydration. Journal of Food Engineering, v. 21, n. 4, p. 513-519, 1994.

7 GALLI, D. C.; BILHALVA, A. B.; RODRIGUES, R. S.; RODRIGUES, L. S. Influência da composição do xarope nas características físico-químicas de pêssegos tipo passa. Revista Brasileira de Agrociência, v.2, n. 3, p. 179-182, 1996.

8 HAWKES, J.; FLINK, J. M. Osmotic concentration of fruit slices prior to freeze dehydration. International Journal of Food Science and Technology, v. 2, p. 265-284, 1978.

9 HENG, K.; GUILBERT, S.; CUQ, J.L. Osmotic dehydration of papaya: influence of process variables on the product quality. Science des Aliments, v. 10, p. 831-848, 1990.

10 IBGE. Instituto Brasileiro de Geografia e Estatística. Produção agrícola municipal e levantamento sistemático da produção agrícola. Nov. 2004. In: BRASIL. Ministério da Agricultura, Pecuária e Abastecimento. Produção Agrícola. Estatísticas: culturas. Disponível em http://www.agricultura.gov.br. Acesso em: 30 ago. 2005.

11 IAL. Instituto Adolfo Lutz. Normas Analíticas do Instituto Adolfo Lutz. 3 ed. São Paulo, 1985. $533 \mathrm{p}$.

12 KOWALSKA, H.; LENART, A. Mass exchange during osmotic pre-treatment of vegetables. Journal of Food Engineering, v. 49, p. 137-140, 2001.

13 KROKIDA, M. K.; KARATHANOS, V. T.; MAROULIS, Z. B.; MARINOSKOURIS, D. Effect of osmotic dehydration on color and sorption characteristics of apple and banana. Drying Technology, v. 18, n. 6, p. 937-950, 2000.

14 MADAMBA, P. S.; LOPEZ, R.I. Optimization of the osmotic dehydration of mango (Mangifera indica I.) slices. Drying Technology, v. 20, p.1227 - 1242, 2002. 
and structural changes in apple (Var. Granny Smith) due to vacuum impregnation with cryoprotectants. Journal of Food Science, v. 63, p. 499-503, 1998.

16 MAVROUDIS, N. E.; GEKAS, V.; SJÖHOLM, I. Osmotic dehydration of apples - effects of agitation and raw materials. Journal of Food Engineering, v. 35, p. 191-209, 1998.

17 MÚJICA-PAZ, H.; VALDEZ-FRAGOSO, A.; LÓPEZ-MALO, A.; PALOU, E.; WELTY-CHANES, J. Impregnation and osmotic dehydration of some fruits: effect of the vacuum pressure and syrup concentration. Journal of Food Technology, v. 57, p. 305-314, 2003a.

18 OZEN, B. F.; DOCK, L.L.; OZDEMIR, M.; FLOROS, J.D. Processing factors affecting the osmotic dehydration of diced green peppers. International Journal of Food Science and Technology, v. 37, n. 5, p. 497-502, 2002.

19 PANAGIOTOU, N. M.; KARATHANOS, V. T.; MAROULIS, Z. B. Effect of osmotic agent on osmotic dehydration of fruits. Drying Technology, v. 17, p. 175-189, 1999.

20 PARK, K. J.; BIN, A.; BROD, F. P. R. Drying of pear d'Anjou with and without osmotic dehydration. Journal of Food Engineering, v. 56, p. 97-103, 2002.

21 PONTING, J. D. Osmotic dehydration of fruits: recent modifications and applications. Process Biochemistry, v. 8, p. 18-20, 1973.

22 RAOULT-WACK, A. L. Advances in osmotic dehydration. Trends in Food Science and Technology, v. 5, p. 255-260, 1994.

23 RASTOGI, N. K.; RAGHAVARAO, K. S. M. S.; NIRANJAN, K.; KNORR, D. Recent developments in osmotic dehydration: methods to enhance mass transfer. Trends in Food Science and Technology, v. 13, p. 4859, 2002.

24 REPPA, A. J.; MANDALA, A. K.; KOSTAROPOULOS; G. D. SARAVACOS. Influence of solute temperature and concentration on the combined osmotic and air drying. Drying Technology, v 17, p. 1449-1458, 1999.

25 SALVATORI, D.; ANDRES, A.; CHIRALT, A; FITO, P. Characterisation of some properties of fruits related to vacuum impregnation. Journal of Food Process Engineering, v. 21, p. 59-73, 1998.

26 SCHIFFMANN, R. F. Microwave process for the food industry. In: HANDBOOK of microwave technology for food applications. Nova York: Marcel Dekker, 2001. p. 299-338.

27 SCHULTZ E. L.; MAZZUCO M. M., MACHADO R. A. F.; BOLZAN, A.; QUADRI M. B.; QUADRI, M. G. M. Effect of pre-treatments on drying, density and shrinkage of apple slices. Journal of Food Engineering, v.78, p. 1103-1110, 2006.

28 SEBRAE. Serviço Brasileiro de Apoio às Micro e Pequenas Empresas. Classificação das MPEs segundo o número de empregados. Disponível em: http://www.cbcde.org.br/pt/noticia/noticia.php. Acesso em:15 mar. 2005.

$29 \mathrm{SHI}, \mathrm{X}$. Q.; FITO, P. Mass transfer in vacuum osmotic dehydration of fruits: a mathematical model approach. Lebensmittel-Wissenschaft und-Technologie, v. 27, p. 67-72, 1994.

30 SILVEIRA, E. T. F.; RAHMAN, M. S.; BUCLE, K. A. Osmotic dehydration of pineapple: kinetics and product quality. Food Research International, v. 29, p. 227-233, 1996.

31 SUMNU, G.; TURABI, E.; OZTOP, M. Drying of carrots in microwave and halogen lamp-microwave combination ovens. Lebensmittel-Wissenschaft und- Technologie, v. 38, p. 549-553, 2005.

32 TALENS, P.; MARTÍNEZ-NAVARRETE, N.; FITO, P.; CHIRALT, A. Changes in optical and mechanical properties during osmodehydrofreezing of kiwi fruit. Innovative Food Science and Emerging Technologies, v. 3 , p. 191-199, 2002.

33 TALENS, P.; ESCRICHE, I.; MARTÍNEZ-NAVARRETE, N.; CHIRALT, A. Influence of osmotic dehydration and freezing on the volatile profile of kiwi fruit. Food Research International, v. 36, p. 635-642, 2003.

34 TORREGGIANI, D.; BERTOLO, G. Osmotic pre-treatments in fruit processing: chemical, physical and 
structural effects. Journal of Food Engineering, Oxford, v.49, p.247-253, 2001.

35 VAN ARSDEL, W.B.; COPLEY, M. J.; MORGAN, A. I. Food Dehydration. Westport: AVI Publishing, 1973. $2 \mathrm{~V}$.

36 VIAL, C.; GUILBERT, S.; CUQ, J. L. Osmotic dehydration of kiwifruits: influence of process variables on the color and ascorbic acid content. Science des Aliments, v. 11, p. 63-67, 1991.

37 VINCENT, J. F. V. Relationship between density and stiffness of apple flesh. Journal of the Science of Food and Agriculture, v. 47, p. 443-462, 1989.

38 ZOZULEVICH, B. V.; D'YACHENKO, E. N. Osmotic dehydration of fruit. Konservn. Ovoshchesush. Prom., v. 7, p. 32-42, 1969. 